\title{
Una experiencia didáctica en la formación de personas jóvenes científicas a través de la participación en investigación científica en el Laboratorio de Física Médica Computacional de la Universidad de Costa Rica
}

Porras-Chaverri, Mariela A.

Una experiencia didáctica en la formación de personas jóvenes científicas a través de la participación en investigación científica en el Laboratorio de Física Médica Computacional de la Universidad de Costa Rica Revista Educación, vol. 46, núm. 1, 2022

Universidad de Costa Rica, Costa Rica

Disponible en: https://www.redalyc.org/articulo.oa?id=44068165003 DOl: https://doi.org/10.15517/revedu.v46i1.43547

\section{(c) 1 (1)}

Esta obra está bajo una Licencia Creative Commons Atribución-NoComercial-SinDerivar 3.0 Internacional. 


\title{
Una experiencia didáctica en la formación de personas jóvenes científicas a través de la participación en investigación científica en el Laboratorio de Física Médica Computacional de la Universidad de Costa Rica
}

\author{
Didactic Experiences for Scientific Research Training of Young Scientists at the University of Costa Rica's \\ Computational Medical Physics Lab
}

Mariela A. Porras-Chaverri

Universidad de Costa Rica, Costa Rica

mariela.porras@ucr.ac.cr

(iD https://orcid.org/0000-0002-0537-2204
DOI: https://doi.org/10.15517/revedu.v46i1.43547 Redalyc: https://www.redalyc.org/articulo.oa? $\mathrm{id}=44068165003$

Recepción: 30 Agosto 2020 Aprobación: 10 Diciembre 2020

\section{Resumen:}

La formación de futuras personas profesionales científicas requiere de oportunidades para el desarrollo de habilidades para la vida (habilidades blandas), con el fin de que puedan llevar a cabo de manera satisfactoria sus labores profesionales; esto es aún más crucial en áreas interdisciplinarias como la Física Médica. Uno de los proyectos de Acción Social de la Universidad de Costa Rica ofrece este tipo de oportunidades a personas estudiantes interesadas en este campo, lo hace mediante mentorías enmarcadas en la participación en proyectos de investigación. Al tratarse de un área de estudio de nivel avanzado y multidisciplinar, se utilizan recursos didácticos lúdicos, que faciliten la comprensión y el disfrute de las actividades en el estudiantado de pregrado. Además, este último aspecto tiene la intención de construir una comunidad de conocimiento alrededor de este interés común. En este trabajo se presentan las experiencias y aprendizajes a partir de las percepciones de las personas participantes en dichas experiencias. Se solicitó al grupo de participantes ( 9 estudiantes y encargada del laboratorio) que brindaran por escrito sus impresiones sobre la experiencia en el laboratorio y con las otras personas participantes, tanto positivas como negativas. Las experiencias de investigación tuvieron como resultado la participación de dos estudiantes como ponentes dentro de un congreso científico internacional. Se concluye de esta experiencia de proyecto que el uso de materiales didácticos para la discusión de conceptos y resultados de investigación científica es un elemento que facilita la participación significativa del estudiantado de pregrado dentro de la investigación, además de que estos espacios de mentoría, empáticos y lúdicos, fomentan el desarrollo de habilidades blandas y la discusión de temas adyacentes a la labor técnica-científica.

Palabras clave: Formación de personal científico, Aprendizaje activo, Desarrollo de las habilidades, Juego creativo.

\section{ABstract:}

One prerequisite for future scientists involves life skills training for successful performance of job duties. The development of soft skills is even more crucial for interdisciplinary areas such as Medical Physics. The University of Costa Rica provides such an opportunity through their Social Outreach Mentoring Program for undergraduate students interested in attaining research experience. Ludic teaching resources are used to cultivate understanding and enjoyment of this area of study, given its advanced and multidisciplinary nature as well as develop a community of knowledge pertaining to this common interest. Varying student experience and learning based on perceptions from 9 participants including the lab administrator are covered who were asked to share their written both positive and negative impressions of their experiences in the lab with their fellow students. As a result, two undergraduate program participants were selected to speak at an international science conference. Such intellectually challenging activities juxtaposed with an empathetic work environment serve to foster student self-confidence and actively engage students. Didactic materials helped enliven discussion and Students displayed more willingness to share their research, which revealed the high number of undergraduates taking part in research programs. Such mentoring opportunities tap into student empathy and help nurture soft skill development through enjoyable learning experiences as well as foster technical and scientific skills required for future employment.

KeYWoRDS: Scientific Personnel Training, Activity Learning, Skills Development, Creative Game. 


\section{INTRODUCCIÓN}

Este trabajo presenta las experiencias vividas en el marco del proyecto de Acción Social ED-3337, que tiene dentro de sus actividades la mentoría a personas estudiantes universitarias de pregrado en Física y con interés en Física Médica en el Laboratorio de Física Médica Computacional (FIMEC) de la Universidad de Costa Rica (UCR). En las experiencias presentadas en este artículo se combina tanto la Acción Social como la Docencia en el contexto de la formación en futuras personas científicas en un laboratorio de investigación.

Este artículo tiene como objetivo presentar una sistematización de las experiencias, tanto didácticas como personales, de las personas participantes de este proyecto y que son integrantes de un laboratorio de investigación científica.

Dado que el trabajo en laboratorios de investigación no forma parte de la formación de estudiantes de pregrado, las experiencias presentadas en este artículo constituyen un insumo para otros laboratorios de investigación en países en desarrollo que deseen incorporar a esta población estudiantil dentro de sus labores y como parte de la formación de futuros científicos y científicas.

Como se desarrolla más adelante en este artículo, para que el estudiantado participe activamente de la investigación científica en el laboratorio, se le debe brindar conocimientos en diversas áreas. Este reto conlleva a la utilización de recursos y estrategias didácticas flexibles e innovadoras en el contexto de entrenamiento para investigación científica en un laboratorio.

\section{MARCO TEÓRICO}

La formación de futuras personas científicas involucra el desarrollo de conocimientos técnicos, habilidades de pensamiento crítico, comunicación y otras habilidades blandas (Bosch y Casadevall, 2017; Kondo y Fair, 2017; Sarkar, Overton, Thompson y Rayner, 2016; Shin y Park, 2020).

En el contexto de la formación en Física, durante la etapa de estudios de pregrado la población estudiantil desarrolla sus conocimientos técnicos mediante cursos teóricos, que son complementados con cursos de laboratorio. Las oportunidades de formar parte de laboratorios de investigación científica y participar activamente en investigación científica publicable son limitadas para estudiantes de pregrado.

Una de las limitaciones para incorporar a estudiantes de pregrado en las labores de investigación científica se debe a que se requiere que el estudiantado investigador tenga conocimientos avanzados en el área de investigación correspondiente. En los casos en que estudiantes de pregrado participan de investigación científica lo hacen de manera superficial, en actividades rutinarias determinadas por el profesorado investigador (Brew y Mantai, 2017; Shaw, Sigmann y Richard, 2020).

El resultado es que, típicamente, el desarrollo de habilidades blandas relacionadas a la investigación científica se inicia hasta la etapa de investigación de tesis de maestría o doctorado. Llegado ese momento, el estudiantado ya ha concluido los cursos del plan de estudios, por tanto, cuenta con el conocimiento teórico necesario para llevar a cabo sus labores de investigación. Sin embargo, participar en experiencias de investigación desde etapas tempranas del pregrado permite que la población estudiantil alcance esta etapa durante el posgrado, con un desarrollo previo de estas habilidades blandas (McKinsey, 2016; Wylie y Gorman, 2018).

Asimismo, formar parte de un grupo de laboratorio y recibir mentoría de parte de investigadores tiene un efecto motivador para el estudiantado, pues también le aumenta la confianza en sus propias capacidades. De esta manera, la población estudiantil va convirtiéndose en un miembro empoderado de la comunidad científica, es decir, en personas capaces de defender sus propias ideas ante otros miembros de esta comunidad (Adams, 2019; Page y Czuba, 1999).

En el caso de futuros y futuras profesionales en Física Médica, que deberán trabajar en ambientes multidisciplinarios y de alta responsabilidad, el desarrollo de habilidades blandas, como la comunicación oral, 
el trabajo en grupo y el manejo del tiempo y recursos, resulta crucial (Prisciandaro et al., 2013 y Gress et al., 2017). Por esta razón, brindar oportunidades para participar en investigación científica a estudiantes de pregrado en Física, que hayan manifestado interés en continuar estudios en Física Médica, permite que el estudiantado de pregrado desarrolle habilidades blandas fundamentales para su futura labor profesional.

De igual manera, el desarrollo de trabajo colaborativo entre estudiantes promueve la conformación espontánea de comunidades de aprendizaje. Al pertenecer a una comunidad de aprendizaje se refuerzan los vínculos entre estudiantes para la transferencia de conocimiento y apoyo emocional. Esto fomenta tanto la retención en la educación superior como el alcance de las metas de formación (Romanoff, 2016).

En el caso de profesionales en Física Médica, la retención del estudiantado interesado es de particular importancia en las instituciones latinoamericanas debido al faltante de recurso humano en esta área para atender las necesidades a nivel hospitalario (Tsapaki, Tabakov y Rehani, 2018).

\section{Metodología}

\section{Laboratorio de Física Médica Computacional}

El Laboratorio de Física Médica Computacional del Centro de Investigación en Ciencias Atómicas, Nucleares y Moleculares (CICANUM) de la Universidad de Costa Rica realiza investigaciones enfocadas en la utilización de métodos computacionales en varias áreas, entre ellas el análisis cuantitativo de imágenes médicas y la simulación de transporte de radiaciones ionizantes. Fue fundado en 2019 y tiene sus raíces en un proyecto de investigación iniciado en 2017 en CICANUM.

Las investigaciones realizadas en FIMEC involucran una diversidad de conocimientos, entre ellos los principios físicos de la interacción de la radiación ionizante con la materia, conceptos de estadística y estimadores estadísticos, el desarrollo y uso de programas computacionales en varios lenguajes de programación (Fortran, $\mathrm{C}++$, Matlab y otros), así como conocimientos básicos de anatomía y fisiología humana.

Estos conocimientos no están presentes en la población de estudiantes típica dentro del laboratorio, sino que deben ser facilitados por la persona investigadora principal a lo largo del desarrollo de los proyectos.

En el trabajo del laboratorio se tiene presente la idea de que, al involucrar a estudiantes de pregrado en la investigación científica, se potencia el desarrollo de las habilidades blandas en cada estudiante. Asimismo, brinda un espacio de introducción a conocimientos técnicos avanzados previo a los cursos de posgrado. De esta manera, al llegar a la etapa de investigación del posgrado en Física Médica, el estudiantado ya cuenta con experiencia previa como investigadores e investigadoras.

Debido a que la mayoría del conocimiento en esta área de investigación se transmite en inglés, es necesario el dominio de este idioma. Al tratarse de un laboratorio de investigación científica es necesario que las personas estudiantes desarrollen habilidades escritas y orales para la presentación de resultados, así como una buena dinámica de trabajo en grupo.

Las actividades de FIMEC son primordialmente de investigación científica; su fin principal es la generación de conocimiento científico publicable. Sin embargo, también se incorpora la mentoría del estudiantado que lo conforma. Estas actividades de mentoría se enmarcan en el proyecto de Acción Social ED-3337 de la Universidad de Costa Rica, así como de la Red de Intercambio Académico Estudiantil (IAVE), la cual se describe en este mismo artículo (Porras-Chaverri et al., 2021). 


\section{Integrantes de FIMEC}

Al momento de realizar esta sistematización participan 9 estudiantes, dos son mujeres y el resto son hombres. Tres de las personas estudiantes proceden de familias en zona rural. Cinco de las personas estudiantes se encuentran en el rango de edad de 25 a 30 años y cuatro entre 20 y 24 años.

Una de las personas estudiantes se encuentra iniciando su trabajo de Maestría en Física Médica y las restantes son estudiantes de segundo año en adelante de bachillerato de la carrera de Física. Una (M) de las personas integrantes ha estado involucrada desde inicios de 2017 y también es asistente de docencia en un curso de física general impartido por la investigadora principal.

Las demás personas estudiantes están desarrollando trabajos de investigación cortos para algunas de sus clases, o bien, desean aprender sobre alguna de las herramientas que se utilizan en el laboratorio. Esta última población puede variar en cada semestre, sin embargo, ocho de las personas estudiantes han formado parte desde marzo de 2019 y se han mantenido activas dentro del laboratorio desde entonces.

De manera temporal, dentro del laboratorio y durante el período que cubre esta sistematización, se ha contado con la participación de tres pasantes internacionales, específicamente dos de Estados Unidos y una procedente de Alemania. La participación de estos pasantes dentro de las actividades del laboratorio permite al estudiantado nacional la interacción de manera regular con personas de otras culturas, lo que propicia el desarrollo de habilidades de comunicación intercultural.

\section{Problemática}

Uno de los retos principales identificados para que el estudiantado sea capaz de participar activamente de un proyecto de investigación en FIMEC es que debe obtener conocimientos muy especializados y multidisciplinarios.

Un agravante de este problema es que gran parte de los fundamentos teóricos de la investigación en FIMEC se enseñan hasta nivel de posgrado, y que no existen cursos regulares a nivel de pregrado donde se cubran estos fundamentos. En la mayoría de los casos, el estudiantado deberá participar de discusiones y desarrollo de herramientas científicas sin tener conocimiento previo de la materia a tratar. Este es el caso de los temas relacionados a interacciones de radiación con la materia, en particular con materiales presentes en el cuerpo humano; temas de profunda vinculación con el quehacer del laboratorio.

Con estas problemáticas en mente, se realizaron discusiones con el asistente de investigación, que ha estado trabajando desde el 2017, sobre posibles recursos útiles para sobrellevarlas. Se conversó sobre las dificultades encontradas desde 2017, y sobre las áreas donde se precisó apoyo adicional, así como las fuentes de este apoyo (personas, libros, artículos, etc.).

A partir de estas discusiones surgió la iniciativa de contar con materiales educativos tangibles como apoyo al trabajo a desarrollar, en particular durante las discusiones de conceptos, métodos y resultados. Con el uso de este tipo de materiales se busca presentar de manera concreta conceptos e ideas abstractas que se utilizan en el quehacer científico del laboratorio.

\section{Materiales educativos}

Durante el primer semestre de 2019 se adquirieron (mediante fondos personales) dos juegos de bloques de madera (también conocidos como tucos) para utilizarse como herramienta en las sesiones de discusión científica sobre las geometrías a incluir en las simulaciones. Uno de los juegos consta de cubos de distintos colores, lo que permite que puedan ser utilizados en discusiones sobre los diversos materiales que constituyen una imagen médica en 3D. 
También se adquirió un modelo anatómico del cuerpo humano que permite observar los órganos internos más importantes en sus posiciones relativas. Dispersos dentro del espacio de laboratorio también se cuenta con juguetes suaves (conocidos como squishies) con formas del cuerpo humano. Los juguetes han sido recogidos en distintas conferencias científicas, en donde se regalan como anuncio de algún producto médico, y se ofrecen al estudiantado como elementos desestresantes, además de que se utilizan como apoyo en el aprendizaje sobre anatomía. Los materiales didácticos descritos se muestran en Figura 1.

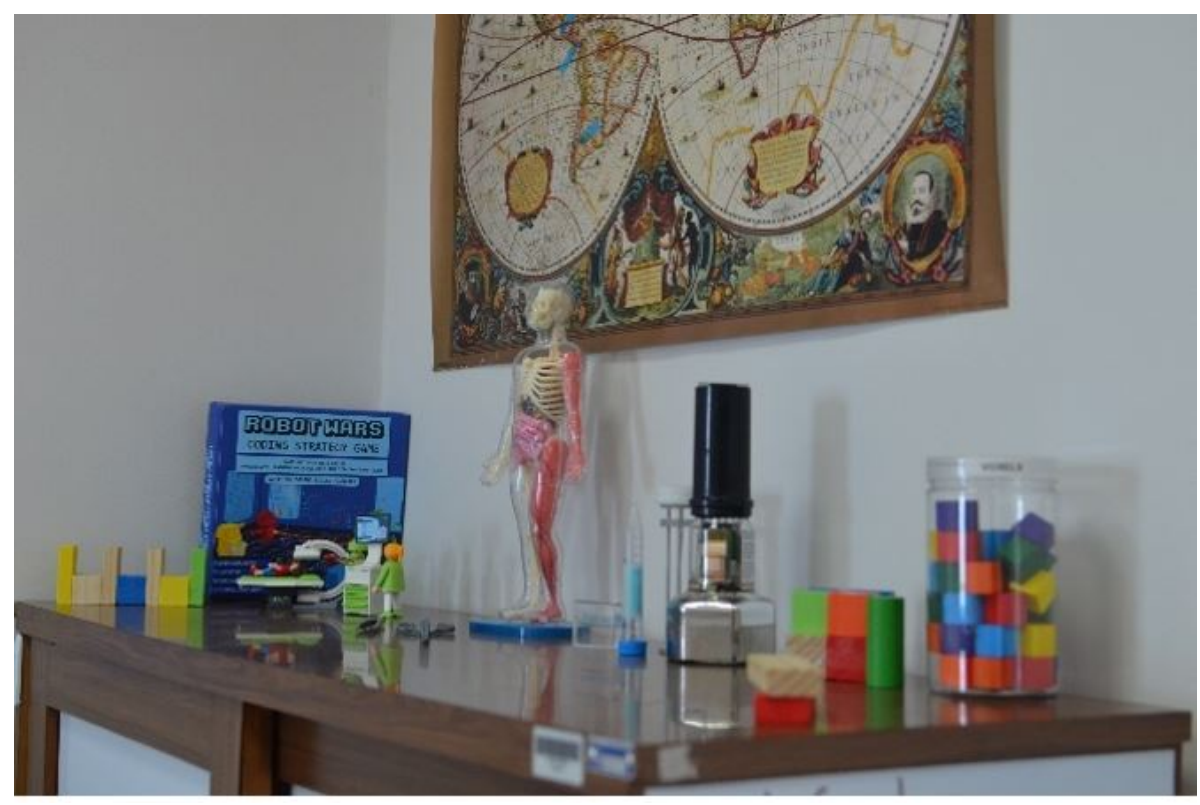

FIGURA 1.

Vista general de materiales didácticos varios.

Fuente: Fotografía tomada por la autora.

Gracias a una donación de un antiguo estudiante (que ya no forma parte del grupo) se cuenta con un implante mamario, que apoya las discusiones sobre propiedades mecánicas de la anatomía de las mamas.

Adicionalmente, y como elemento motivador para las personas estudiantes con menos experiencia en programación computacional, se compró un juego de mesa diseñado para enseñar principios de programación a niños llamado Robot Wars. Este juego se utiliza como material introductorio a conceptos de programación para permitir a nuevas personas estudiantes explorar conceptos de programación sin la presión de que sean parte de un trabajo o una tarea. Adicionalmente, este juego puede utilizarse en actividades sociales como la Casa Abierta u otras organizadas por el grupo. Este juego se muestra en Figura 2. 


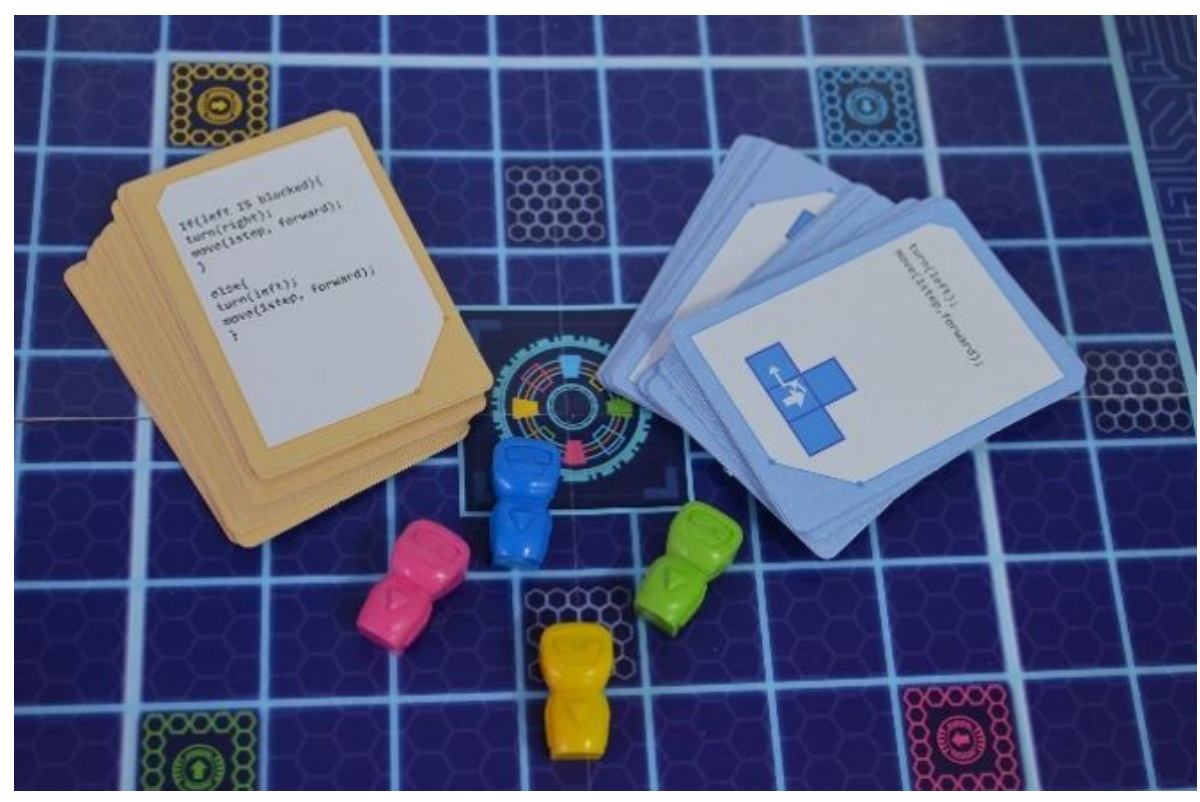

FIGURA 2.

Componentes del juego de mesa Robot Wars.

Fuente: Fotografía tomada por la autora.

Posteriormente, en el segundo semestre de 2019, se adquirió un juego de bingo, del cual la tómbola se utiliza para demostrar algunos conceptos utilizados en simulaciones con métodos de MonteCarlo; particularmente, el concepto de utilizar números aleatorios para seleccionar los parámetros a utilizar para una partícula individual en simulaciones de transporte de radiaciones.

\section{Cuaderno de bitácora}

De manera paralela, a cada una de las personas estudiantes se le da un cuaderno de bitácora, al que, dentro del contexto de FIMEC, se le denomina el Libro Azul (debido al color de su portada). El propósito de este cuaderno es facilitar prácticas de buen manejo de la información, en particular sobre el proceso de aprendizaje y generación de conocimiento durante las reuniones y el trabajo individual, así como de la organización de las tareas que les corresponde desarrollar. De esta manera se busca fomentar el compromiso y el buen uso del tiempo. Un propósito adicional al acto de darles un Libro Azul al estudiantado es que este constituye un pequeño acto de bienvenida al laboratorio, y un elemento en común con otras personas estudiantes que ya forman parte del grupo.

\section{Desarrollo de misión y visión de FIMEC}

De manera paralela a las conversaciones con M, el asistente de investigación, la investigadora principal realizó una reflexión para definir la misión y visión del laboratorio, así como de sus valores principales y otros aspectos organizacionales, de cara al rol del nuevo laboratorio como espacio de formación para jóvenes científicas y científicos. Esta reflexión fue realizada de manera individual por la investigadora, según su rol de encargada del laboratorio dentro de la estructura del CICANUM, tomando en cuenta las experiencias y conversaciones previas con el asistente.

En esta reflexión, se determinó además que el laboratorio estaría orientado por cinco valores principales: respeto, eficiencia, cooperación, compromiso y creatividad-lúdica. Éste último es de particular interés, tanto 
como motivador a nivel emocional del estudiante y como actividad generadora de conocimiento científico en sí y constituyó uno de los puntos considerados como clave dentro del proceso de aprendizaje, tanto por la investigadora principal como por el asistente de investigación.

La definición de una serie de valores que enmarquen las actividades del laboratorio brinda los inicios de una cultura común a sus miembros, y presenta de manera explícita aspectos que se consideran clave para el éxito de los procesos aprendizaje y de investigación científica dentro del laboratorio. De igual manera, muestran de manera explícita aspectos fundamentales para las futuras labores del alumnado, relacionados al desarrollo de habilidades blandas, pero que pueden pasar desapercibidos dentro de la formación universitaria técnica.

En esta etapa, la investigadora principal se apoyó en los conocimientos obtenidos durante un curso de educación en STEM (Ciencia, Tecnología, Ingeniería y Matemáticas, por sus siglas en inglés), se utilizaron metodologías de aprendizaje activo y pensamiento de diseño (Design Thinking) organizado por Sistema Nacional de Acreditación de la Educación Superior (SINAES) y Laspau, así como en sus experiencias previas en distintos grupos de investigación y en los conocimientos obtenidos en un curso de Gestión del Conocimiento del Organismo Internacional de Energía Atómica (OIEA).

\section{Espacio físico del laboratorio}

A raíz de estos análisis, y según las recomendaciones de un investigador de la Escuela de Arquitectura, se procuró que dentro del laboratorio existieran opciones para sentarse de manera individual, en grupos y en posiciones corporales distintas. Este tipo de configuración del espacio fomenta el trabajo colaborativo y el intercambio de conocimientos, al poderse involucrar cada estudiante con otras labores y discusiones que ocurran en el laboratorio. En particular, dentro del laboratorio se tienen distintos tipos de sillas de oficina con rodines, así como dos muebles que funcionan como banqueta, cubiertos por unas pequeñas alfombras de zacate artificial que también pueden ser colocadas en el suelo. Una vista del espacio físico del laboratorio puede observarse en la Figura 3.

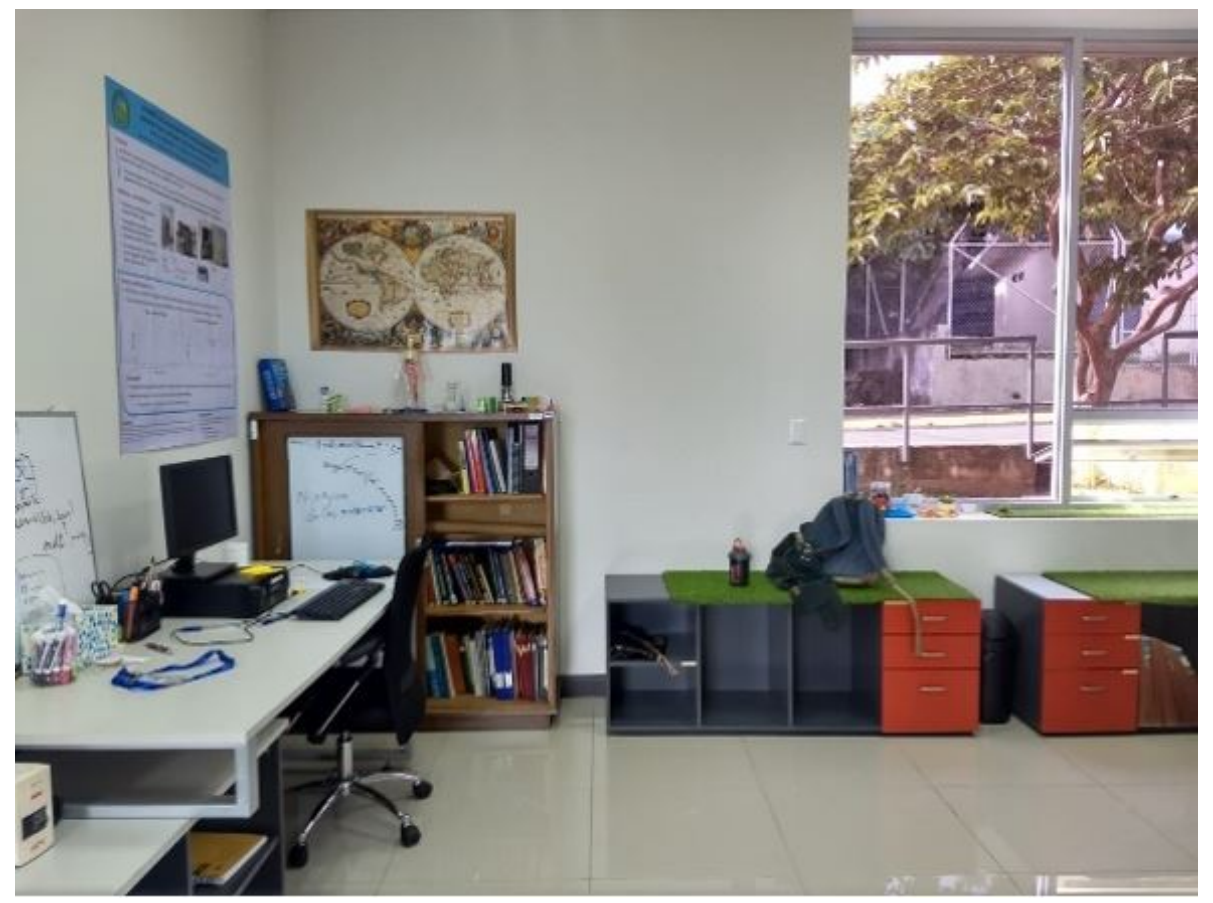

FIGURA 3.

Vista general del espacio físico de FIMEC.

Fuente: Fotografía tomada por la autora. 
Esta configuración permite al alumnado no solo cambiar de posición mientras utilizan el espacio de laboratorio, si no también que fomenta un entorno relajado y una relación menos jerárquica entre estudiantes y la persona investigadora principal, pues no se cuenta con un espacio fijo asignado a ella. Al tener la investigadora principal un rol de apoyo en los procesos de aprendizaje de las personas estudiantes, su lugar de trabajo es al lado de cada estudiante y no en un espacio aislado de trabajo individual.

Una limitante dentro del diseño del espacio es que, debido a limitaciones del presupuesto, se debió trabajar con mobiliario ya existente dentro del CICANUM. Si bien este fue seleccionado según el criterio del arquitecto, no se trata de mobiliario diseñado con el trabajo colaborativo en mente. En particular, las mesas con las que se cuentan no tienen rodines, por lo que no se cambia mucho su configuración durante el uso cotidiano, a pesar de que son livianas y hay suficiente espacio para acomodarlas de manera distinta.

Dentro del espacio de trabajo se cuenta también con pizarras tradicionales en la pared, y superficies de tipo pizarra en el sobre de los escritorios y otras que envuelven algunos muebles; estas superficies constan de pliegos adhesivos de un material sobre el que se puede escribir con marcador. El contar con estas superficies y marcadores de diversos colores fomenta la espontaneidad y claridad en las discusiones científicas. El uso de estas superficies también fue recomendado por una desarrolladora de programas en la empresa Google, quien es una persona conocida de la investigadora principal.

Contar con un espacio visual común permite al estudiantado no solo comprender los conceptos que se les están explicando, si no también exponer sus ideas y el entendimiento que van teniendo sobre estos. Otro beneficio de tener estas pizarras es que se cuenta con la posibilidad de realizar registros de las sesiones de trabajo conjunto, en particular, las personas estudiantes suelen tomar fotografías con sus teléfonos personales.

Por otro lado, el espacio también cuenta con elementos relacionados al quehacer científico. En particular, se cuenta con una tabla periódica y afiches de investigaciones previas, así como un mapa conceptual de los distintos subproyectos, actuales y futuros, desarrollados en el laboratorio. En este mapa se incluye una breve reseña del subproyecto y el nombre de la persona estudiante a cargo, así como las relaciones con otros proyectos del laboratorio.

Uno de los afiches colgados presenta una investigación realizada por uno de los asistentes del laboratorio, con el fin de no solo dar a conocer el trabajo realizado dentro de FIMEC, sino también para mostrar al alumnado lo que se espera de él (expectativas) y lo que puede lograr con su aprendizaje (posibilidades).

Otros elementos presentes en el espacio, y con carácter motivador, incluyen una serie de láminas tomadas de un calendario sobre mujeres en ciencia y un mapa mundial, donde se marcan los países en que miembros o colaboradores del laboratorio han presentado trabajos relacionados a las investigaciones realizadas, incluyendo presentaciones realizadas por estudiantes.

Dentro del espacio también se cuenta con elementos gráficos de la Red IAVE, se incluyen logos y boletines, así como materiales promocionales de otras instituciones que forman parte de IAVE, y un afiche con un resumen del código de ética de una de las instituciones colaboradoras (regalo de una de las personas colaboradoras de FIMEC).

\section{Casa Abierta 2019}

En marzo de 2019 se realizó una Casa Abierta a manera de inauguración del nuevo espacio. En esta actividad se recibieron visitantes externos al laboratorio (estudiantes de bachillerato en Física y en Imagenología). Las personas estudiantes del laboratorio presentaron una reseña de los respectivos trabajos a su cargo.

Como parte de la Casa Abierta se realizaron dinámicas interactivas entre estudiantes y visitantes. Estas actividades incluyeron la creación de una red social en la pared del laboratorio, donde se muestra la relación entre estudiantes y otras instituciones internacionales. Esta red social está aún colocada en la pared para visibilizar el contexto del laboratorio dentro de un entorno global, y el papel que juega cada una de las personas estudiantes. 
La Casa Abierta finalizó con una partida del juego de mesa diseñado para la enseñanza de principios de programación para niños. El objetivo de esta actividad fue mostrar, a posibles personas interesadas en el trabajo del laboratorio, que la programación computacional no es una tarea inaccesible para ellos.

A partir de la actividad de Casa Abierta surgió el interés de tres estudiantes de aprender sobre las técnicas usadas en el laboratorio, dos estudiantes de bachillerato en Física y una persona de Tecnologías en Salud, quien formó parte del laboratorio durante poco tiempo, pues debió enfocarse en su plan de estudios.

Cabe mencionar que esta persona también debió enfrentarse a nuestro desconocimiento de que algunas herramientas no estaban disponibles en la plataforma computacional que utiliza en su casa, por lo que debió invertir mucho tiempo en buscar recursos para ver si le era posible instalarlas.

Se considera que tener que realizar labores de alto nivel de conocimiento técnico en un entorno incierto (al no saber si se podían instalar), y resultar este trabajo infructuoso (al no existir la posibilidad de instalarlas), además de la carga de trabajo académico, contribuyeron a la decisión de salirse del laboratorio. Al momento de anunciar esta decisión, se dejó abierta al estudiante la posibilidad de regresar a colaborar al laboratorio si así lo desea en el futuro.

El resto de estudiantes con interés se ha involucrado en el quehacer del laboratorio a lo largo del año. En uno de los casos fue necesaria una breve conversación con la persona para dejar en claro que no es necesario mayor conocimiento para formar parte del laboratorio.

En el otro caso, la persona estudiante se incorporó al trabajo del laboratorio en julio de 2019. En el segundo semestre de 2019, esta persona involucró a otras dos estudiantes en el quehacer del laboratorio, una de ellas estudiante de Física y otra estudiante de Ciencias Médicas con estudios y experiencia previa en Computación. Al momento de realizar esta reseña estas dos personas estudiantes se encuentran en etapa de inducción y aprendizaje de las herramientas básicas.

\section{Visitantes internacionales}

Entre los meses de mayo y agosto de 2019 se contó con la visita de dos pasantes procedentes de Estados Unidos, uno es estudiante de bachillerato en Ingeniería Computacional, y la otra pasante es estudiante de bachillerato en Economía.

En los meses de agosto a octubre del mismo año también se recibió a una segunda estudiante alemana, cuyo trabajo está enmarcado dentro de uno de los proyectos de investigación del laboratorio. Como parte de su pasantía, presentó una charla sobre el tema de su investigación en una de las actividades de la Red IAVE.

Contar con la presencia de estas personas pasantes dentro del espacio de laboratorio fomentó el uso del inglés como idioma en las discusiones y reuniones del grupo, además de que permitió al estudiantado escuchar acentos y expresiones ajenas a las presentadas en medios de comunicación. Su presencia también les permitió observar la forma de trabajar de personas provenientes de otro entorno cultural.

El estudiantado también pudo interactuar de manera personal con las personas pasantes, sin la presencia de la investigadora principal. Esto tiene como fin fomentar la creación de redes de colaboración y apoyo a nivel internacional, así como el empoderamiento del alumnado como participante activo dentro de un mundo global.

Es de notar que, incluso después de la partida del estudiantado internacional, algunas de las personas estudiantes del grupo, como iniciativa personal y ocasional, han seguido utilizando el idioma inglés para comunicarse con la investigadora principal o con otros estudiantes del grupo, tanto por escrito como oralmente. 


\section{Preparación de envíos al congreso internacional}

Con el fin de empoderar al estudiantado en sus capacidades científicas, se le invitó a presentar un resumen, basado en el trabajo realizado en el laboratorio, al Latin-American Symposium on Nuclear Physics and Applications 2020 (LASNPA 2020); el congreso fue realizado en enero del 2020 en las instalaciones de la UCR. No fue posible invitar a todo el grupo de estudiantes a participar, pues gran parte se encontraba en etapas previas al trabajo científico en sí.

Previo al envío al congreso, la investigadora principal les dio retroalimentación sobre sus resúmenes y sugirió cambios a realizar en ellos.

Al tratarse de un evento internacional que se realizó en el país, constituyó una oportunidad para que no solo enviaran el resumen, sino que también presentaran sus trabajos.

Los trabajos enviados por las personas estudiantes pasaron por el mismo proceso de revisión por pares científicos que los trabajos enviados por profesionales del área, pues este congreso no cuenta con participación diferenciada para estudiantes.

\section{Perspectivas de las personas integrantes respecto a las experiencias en el laboratorio}

Como parte de esta sistematización, se realizó una recopilación de las opiniones del estudiantado con respecto a su experiencia dentro del laboratorio. Esta recopilación tuvo como fin evaluar de manera cualitativa la experiencia global de las personas estudiantes pertenecientes a este laboratorio mediante sus percepciones de las experiencias vividas. Esta recopilación de experiencias se realizó previo al envío de las ponencias al LASNPA 2020.

Se le solicitó que brindara sus impresiones sobre el laboratorio, relación con sus pares, dinámicas de trabajo, si consideran que la experiencia le puede ayudar en su carrera o en su vida, y que consideraran aspectos técnicos y emocionales, incluyendo empoderamiento, habilidades blandas y juego. Se le indicó también que incluir aspectos negativos dentro de sus comentarios sería de utilidad para el análisis de la experiencia a sistematizar. Las personas estudiantes fueron informadas de que sus opiniones se utilizarían en esta sistematización de manera anónima y se les dio la opción de no brindar sus opiniones si no lo deseaban.

\section{Resultados}

\section{Experiencias de presentación de ponencias en congreso internacional}

De las personas estudiantes invitadas, tres enviaron resúmenes escritos por ellas (dos en un mismo trabajo). Un tercer resumen fue enviado a partir del trabajo realizado con la pasante alemana en 2018; gracias a este, una de las personas estudiantes tuvo la oportunidad de enviar dos ponencias a este congreso.

Dos de los trabajos fueron presentados como ponencia oral por las personas estudiantes autoras de las ponencias, y el trabajo realizado por la pasante alemana fue presentado como ponencia oral por la persona estudiante segunda autora. De esta manera, una de las personas estudiantes realizó dos presentaciones dentro de este congreso.

El que personas estudiantes de pregrado hayan logrado desarrollar sus proyectos de investigación, de manera que fueran aceptados en un evento científico internacional con revisión de pares científicos, se toma como evidencia de la calidad del trabajo realizado. Los resúmenes enviados pusieron en evidencia el desarrollo de sus habilidades de comunicación escrita en inglés.

Asimismo, las personas estudiantes mostraron el desarrollo de sus habilidades orales en la presentación de sus trabajos en idioma inglés, y al responder preguntas del público en este idioma durante la conferencia. 
Estos resultados muestran que, a través de las experiencias presentadas en este documento, el estudiantado no solo tiene la oportunidad de desarrollar sus habilidades, sino que también lo hace mediante el desarrollo de trabajos científicos de calidad.

La participación en este congreso tiene además un efecto empoderador en las personas estudiantes que presentaron, así como en sus colegas, ya que no solo fue un reto para quienes participaron, superado con creces, sino que también muestra a las demás personas lo que se puede lograr.

\section{Perspectivas de las personas participantes}

De los comentarios del estudiantado se interpreta que la experiencia dentro del laboratorio ha sido positiva para todas las personas participantes, tanto para quienes han formado parte del laboratorio desde un inicio, como para quienes recién se incorporan.

Varios de los testimonios hacen referencia al aprendizaje de conocimientos técnicos nuevos, como conocimientos sobre temas de salud y computación. De algunos comentarios se puede deducir que la experiencia dentro del laboratorio permitió adquirir nuevos conocimientos que les ha brindado una perspectiva crítica sobre las limitaciones de su formación profesional. Darse cuenta de estas limitaciones ha inducido al estudiantado a identificar áreas de mejora en su formación y a encontrar maneras para llenar esos vacíos del conocimiento.

Por otro lado, algunos de los comentarios brindados mencionan que las experiencias vividas han sido útiles en el descubrimiento y aprendizaje de nuevas habilidades, incluyendo el caso de habilidades de comunicación y de trabajo en equipo.

Es de destacar que varias personas estudiantes mencionan conceptos como el disfrute por la Ciencia, la creatividad y la libertad de exploración de saberes y habilidades, a pesar de que pocas mencionaron de manera explícita el juego, o incluso lo interpretan como algo ajeno a las prioridades del laboratorio. En todos estos aspectos está presente el juego creativo como una componente esencial para el desarrollo de habilidades, tanto de habilidades para la vida (habilidades blandas) como de conocimientos técnico-científicos (habilidades duras). En este sentido, la perspectiva de la experiencia del estudiantado contrasta con la de la investigadora principal, pues para ella ese aspecto lúdico está muy vinculado a las labores del laboratorio.

Como un aspecto relacionado a estas interacciones más lúdicas, al menos una persona estudiante expresó interés en socializar más allá de las reuniones de trabajo para contar con un espacio donde compartir vivencias de otros aspectos de la vida universitaria.

Un caso de particular interés de los testimonios corresponde al de la persona estudiante con mayor trayectoria dentro del laboratorio, quien debió enfrentarse al aprendizaje inicial de muchas herramientas técnicas al mismo tiempo que la investigadora principal, por lo que no contó con muchos de los apoyos didácticos y sociales con los que han contado estudiantes que se han incorporado más recientemente. Además, ha debido llevar a cabo labores de mayor responsabilidad, como entrenar a estudiantado nuevo y desarrollar programas de computación que otros y otras estudiantes usarán, así como ser partícipe activo del proceso del diseño didáctico del laboratorio.

Asimismo, la trayectoria de esta persona estudiante en el área de docencia hace que, como partícipe del proceso, tenga una visión mucho más orientada a objetivos, tanto del trabajo como del aprendizaje, y de las metas profesionales.

Su testimonio provee una ventana hacia el posible desarrollo futuro del estudiantado y al tipo de experiencias que podrían tener mayor impacto en la formación de este. En particular, este testimonio muestra que las experiencias dentro del laboratorio han permitido el desarrollo de la resiliencia ante los retos, una habilidad fundamental para el desarrollo exitoso de proyectos de largo plazo y una vida laboral plena. 
Un aspecto común que se deduce de los comentarios es que todas las personas estudiantes han aumentado la confianza en sus capacidades, por lo que la experiencia dentro del laboratorio ha sido útil dentro de su proceso de empoderamiento.

Un caso interesante es que, dentro del contexto de ciencias, el concepto de empoderamiento está muy ligado a grupos sociales con menor representación. En este contexto no sorprende que uno de los estudiantes (de género masculino) vincule el concepto de empoderamiento de las estudiantes (de género femenino) dentro de la ciencia y no lo relacione con su propio proceso.

Queda la interrogante de si este proceso de empoderamiento continuará más allá del laboratorio y si se transmitirá a otras áreas de su vida personal y profesional. Algunos testimonios sugieren que este podría ser el caso, pero no se puede saber con certeza aún. En un futuro, cuando el estudiantado se gradúe y se incorpore a la vida laboral, podría ser importante preguntarle si estas experiencias le han sido de utilidad en su trabajo profesional.

Si bien parte de las personas estudiantes mencionaron aspectos negativos en su experiencia (como la falta de acceso al espacio físico, cansancio y desmotivación ante trabajos repetitivos), se debe mencionar una limitación de los testimonios recibidos, ya que, al ser recopilados directamente por la investigadora principal, el grupo de participantes se pudo haber concentrado únicamente en los aspectos positivos, o en los aspectos negativos de menor impacto. En ese sentido, es importante mantener los canales de comunicación abiertos entre las personas estudiantes y la investigadora, y, de ser posible en el futuro, instaurar espacios para recibir retroalimentación de manera anónima.

\section{Conclusiones}

De esta labor de sistematización se desprenden algunos aprendizajes de particular interés.

Por un lado, que estudiantes de pregrado son capaces de realizar trabajo científico en áreas avanzadas, e incluso proponer sus propias ideas; esto si cuentan con las herramientas educativas y el apoyo para llevarlo a cabo. En este sentido, la utilización de materiales educativos apropiados ha sido un elemento clave en el éxito de la transmisión del conocimiento avanzado necesario para el desarrollo de las investigaciones científicas.

Por otro lado, al crearse intencionadamente un entorno de trabajo ameno y colaborativo, es posible fomentar el empoderamiento del estudiantado y la formación de una red social de apoyo. El alumnado se siente en capacidad de proponer ideas y de hacerle frente a los retos que se proponen. Asimismo, se siente en confianza de compartir, entre sí y con la persona docente, las experiencias en su quehacer académico y de vida en general, tanto positivas como negativas. Esto tiene como consecuencia no solo estudiantes con más seguridad de sus habilidades, sino también más resilientes a los retos emocionales que conlleva el trabajo científico.

Otras actividades por realizar corresponden a espacios de interacción social fuera de las reuniones de trabajo. Organizar actividades sociales opcionales, como lo puede ser un café-tertulia o una visita a algún museo, provee oportunidades para el desarrollo de una cultura común y lazos emocionales entre las personas estudiantes. De igual manera, son una oportunidad de vincular el trabajo del laboratorio con otras actividades sociales y de poner en contexto su trabajo, lo que tendría un rol positivo en la autoestima del estudiantado, al cumplir un rol dentro de una actividad que tiene beneficio a la sociedad.

Finalmente, a pesar de ser esta la primera iteración de estudiantes que forma parte de esta experiencia, es importante mantener este tipo de vínculos a través de las distintas generaciones de estudiantes. Con este fin, las redes sociales podrían utilizarse de manera más proactiva, con el fin de establecer espacios donde puedan interactuar tanto estudiantes actuales como exestudiantes.

A partir de esta experiencia, es claro que un entorno que fomente el juego, ejercicios creativos entre el estudiantado y que utilice herramientas lúdicas para el aprendizaje, tiene consecuencias positivas dentro del 
Mariela A. Porras-Chaverri. Una experiencia didáctica en la formación de Personas jóvenes científi...

desarrollo integral las personas estudiantes como futuras profesionales, así como para la adquisición de los conocimientos técnicos y habilidades necesarias para su trabajo en investigaciones científicas.

\section{Referencias BIBLIOGRÁficAS}

Adams, S. K. (2019). Empowering and motivating undergraduate students through the process of developing publishable research[Empoderar y motivar a los estudiantes de pregrado a través del proceso de desarrollo de investigaciones publicables].Frontiers in Psychology, 10 https://doi.org/10.3389/fpsyg.2019.01007

Bosch, G., y Casadevall, A. (2017). Graduate biomedical science education needs a new philosophy [La educación de posgrado en ciencias biomédicas necesita una nueva filosofía].MBio, 8(6). https://doi.org/10.1128/mbio.0153 9-17

Brew, A., y Mantai, L. (2017). Academics' perceptions of the challenges and barriers to implementing research-based experiences for undergraduates[Percepciones de los académicos sobre los desafíos y barreras para implementar experiencias basadas en la investigación para estudiantes universitarios].Teaching in Higher Education, 22(5), 551-568. https://doi.org/10.1080/13562517.2016.1273216

Gress, D., Jordan, D., Butler, P., Clements, J., Coleman, K., Goff, D., Martin, M., Nishino, T., Pizzutiello, R., Wagner, L. y Fairobent, L. (2017). An updated description of the professional practice of diagnostic and imaging medical physics: the report of AAPM diagnostic work and workforce study subcommittee [Una descripción actualizada de la práctica profesional de la física médica de diagnóstico e imágenes: informe del subcomité de estudio de la fuerza laboral y el trabajo de diagnóstico de la AAPM]. College Park, MD: American Association of Physicists in Medicine. $\mathrm{h}$ ttps://doi.org/10.37206/163

Kondo, A. E., y Fair, J. D. (2017). Insight into the chemistry skills gap: the duality between expected and desired skills [Información sobre la brecha de habilidades químicas: la dualidad entre habilidades esperadas y deseadas]. Journal of Chemical Education, 94(3), 304-310. https://doi.org/10.1021/acs.jchemed.6b00566

McKinsey, E. (2016). Faculty mentoring undergraduates: The nature, development, and benefits of mentoring relationships[Profesorado dando mentoría a estudiantes universitarios: la naturaleza, el desarrollo y los beneficios de las relaciones de mentoría. Investigación de enseñanza y aprendizaje].Teaching \& learning inquiry The ISSOTL Journal, 4(1). https://doi.org/10.20343/teachlearninqu.4.1.5

Page, N., y Czuba, C.E. (1999). Empowerment: What Is It? [Empoderamiento: ¿Qué es?].The Journal of Extension, 37(5). https://archives.joe.org/joe/1999october/comm1.php

Porras-Chaverri, M. A., Padilla, L., Alvarez, D., y Rosado-Méndez, I. (2021). International experiences on a budget: The role of online technologies in the training of young scientists in developing countries [Experiencias internacionales con un presupuesto: el papel de las tecnologías en línea en la formación de científicos jóvenes en los países en desarrollo].Proceedings of the XVI Mexican Symposium on Medical Physics, (pp. 040013-1040013-6). https://doi.org/10.1063/5.0051229

Prisciandaro, J., Willis, C., Burmeister, J., Clarke, G., Das, R., Esthappan, J., Gerbi, B., Harkness, B., Patton, J., Peck, D., Pizzutiello, R., Sandison, G., White, S., Wichman, B., Ibbott, G., y Both, S. (2013). Essentials and guidelines for clinical medical physics residency training programs [Fundamentos y directrices para los programas de formación de residencia en física médica clinica]. (pp. 1-12), College Park, MD: American Association of Physicists in Medicine. https://doi.org/10.37206/149

Romanoff, S. J. (2016). The undergraduate learning community: A bridge to understanding why[La comunidad de aprendizaje de pregrado: un puente para comprender por qué].New directions for teaching and learning, 2016(145), 77-84. https://doi.org/10.1002/tl.20177

Sarkar, M., Overton, T., Thompson, C., y Rayner, G. (2016). Graduate employability: views of recent science graduates and employers [Empleabilidad de los graduados: opiniones de los recién graduados en ciencias y empleadores].International Journal of Innovation in Science and Mathematics Education, 24(3), 31-48. https:// openjournals.library.sydney.edu.au/index.php/CAL/article/view/11043/10644 
Shaw, N. N., Sigmann, S. B., y Richard, L. B. (2020). The research storyboard: ideas for cultivating safe, engaged, and empowered undergraduate research students [El guión gráfico de investigación: ideas para cultivar estudiantes de investigación universitarios seguros, comprometidos y empoderados]. Journal of Chemical Education, 98(1),167-174. https://doi.org/10.1021/acs.jchemed.0c00097

Shin, W. y Park, J. (2020). Developing a list of behavioral characteristics of creative physicists during their growth period[Desarrollar una lista de características de comportamiento de los físicos creativos durante su período de crecimiento]. International Journal of Science and Mathematics Education, 19(4), 701-725. https://doi.org/10. 1007/s10763-020-10082-w

Tsapaki, V., Tabakov, S. y Rehani, M. M. (2018). Medical physics workforce: A global perspective[Fuerza laboral de física médica: una perspectiva global]. Physica Medica, 55, 33-39. https://doi.org/10.1016/j.ejmp.2018.10.012

Wylie, C. D. y Gorman, M. E. (2018). Learning in laboratories: how undergraduates participate in engineering research [Aprendizaje en laboratorios: cómo participan los estudiantes universitarios en la investigación en ingeniería]. American Society for Engineering Education Annual Conference and Exposition. https://www.as ee.org/public/conferences/106/papers/22448/view

\section{INFORMACIÓN ADICIONAL}

Cómo citar: Porras-Chaverri, M. A. (2022). Una experiencia didáctica en la formación de personas jóvenes científicas a través de la participación en investigación científica en el Laboratorio de Física Médica Computacional de la Universidad de Costa Rica. Revista Educación, 46(1). Recuperado de http://doi.org/ $10.15517 /$ revedu.v46i1.43547 